\title{
Kinetic theory of non-hamiltonian statistical ensembles
}

\author{
A.V.Zhukov ${ }^{1,2}$, J.Cao $^{1}$ \\ ${ }^{1}$ Department of Chemistry, Massachusetts Institute of Technology, Cambridge, MA 02139, USA \\ 2 Department of Chemistry, University of Western Ontario, London, Ontario, N6A 5B7, Canada
}

Received September 21, 2005

\begin{abstract}
A nonequilibrium statistical operator method is developed for ensembles of particles obeying non-Hamiltonian equations of motion in classical phase space. The main consequences of non-zero compressibility of phase space are examined in terms of time-dependent dynamic quantities. The generalized transport equations involve the phase-space compressibility in a non-trivial way. Our results are useful in molecular dynamics simulation studies as well as nonequilibrium or quasiclassical approximations of quantum-classical dynamics.
\end{abstract}

Key words: non-Hamiltonian systems, nonequilibrium statistical operator, Zubarev formalism, molecular dynamics

PACS: $05.20 .-y, 05.20 . G g, 82.20 .-w$

Theoretical interest in the non-Hamiltonian motion appears in the early works [1-3], where it was realized that the constant-temperature simulations require the introduction of additional non-Hamiltonian acceleration into dynamical equations. This has opened new research directions associated with extended systems. For the past few years the physics of non-Hamiltonian systems was partially developed for molecular dynamics simulation purposes [4-7,9]. Particular applications of non-Hamiltonian dynamical systems are overviewed in a recent paper [10], where the authors exploit their formulation of non-Hamiltonian statistical mechanics [11]. In this regard it is also important to mention the interesting studies of mixed quantum-classical systems [12-16], where the non-Hamiltonian behavior plays an essential role.

One of the most popular non-Hamiltonian ensembles is the so-called Nosé-Hoover chain [6]. In case of two thermostat variables, the corresponding Hamiltonian is

$$
\mathcal{H}_{\mathrm{NH}}=\frac{p^{2}}{2 m}+\frac{p_{\eta_{1}}^{2}}{2 M_{\eta_{1}}}+\frac{p_{\eta_{2}}^{2}}{2 M_{\eta_{2}}}+V(q)+g k_{b} T\left(\eta_{1}+\eta_{2}\right),
$$

where $\eta_{i}$ and $p_{\eta_{i}}$ are the conjugate thermostat variables, $g$ is the number of degrees of freedom, $T$ is the thermostat temperature. It can be shown that the Hamiltonian (1) is conserved by the following (non-Hamiltonian) equations of motion:

$$
\begin{aligned}
\dot{q} & =\frac{p}{m}, \\
\dot{\eta}_{i} & =\frac{p_{\eta_{i}}}{M_{\eta_{i}}}, \\
\dot{p} & =-\frac{p_{\eta_{1}}}{M_{\eta_{1}}} p-\frac{\partial V(q)}{\partial q}, \\
\dot{p}_{\eta_{1}} & =\frac{p^{2}}{m}-g k_{b} T-p_{\eta_{1}} \frac{p_{\eta_{2}}}{M_{\eta_{2}}}, \\
\dot{p}_{\eta_{2}} & =\frac{p_{\eta_{1}}^{2}}{M_{\eta_{1}}}-g k_{b} T .
\end{aligned}
$$

In real MD simulations the particular choice of non-Hamiltonian ensemble can vary depending on the physical problem under study. But all of them can be described in general formalism we present 
here. Recently using a generalization of the symplectic form of the Hamilton equations of motion the authors of [17] showed that there is a unique general structure that underlies the majority of the equations of motion for extended systems. Furthermore, Sergi $[18,25]$ proposed a consistent theory of statistical mechanics for non-Hamiltonian systems under equilibrium conditions. The tempting feature of the theory $[17,18,25]$ is that it is based on the introduction of a suitable Poisson bracket, and has the general structure of classical formalism [19] with a natural appearance of the nonzero phase-space compressibility. The main feature of the systems of interest is energy conservation, which is a reason for non-Hamiltonian behavior. Therefore it is interesting to consider the situation, when the energy is still conserved, but the system itself is out of equilibrium, particularly when the dynamical quantities explicitly depend on time.

The starting point in describing the non-Hamiltonian statistical ensembles is the introduction of the proper non-Hamiltonian Poisson bracket:

$$
\{A(t), C(t)\}=\sum_{i k}^{2 N} \frac{\partial A}{\partial x_{i}} \mathcal{B}_{i k} \frac{\partial C}{\partial x_{k}},
$$

where $\mathbf{x}=(q, p)=\left(q_{1}, q_{2} \ldots q_{N} ; p_{1}, p_{2} \ldots p_{N}\right)$ is the $2 N$-dimensional vector in phase space, $A(t)$ and $C(t)$ are arbitrary dynamical quantities (we allow them to depend on time explicitly), $\mathcal{B}_{i k}=-\mathcal{B}_{k i}$ is an antisymmetric matrix, whose elements are functions of $\mathbf{x}$ and, possibly, time. Following [18] we write the equations of motion in standard form $\dot{x}_{j}=\left\{x_{j}, \mathcal{H}\right\}$, where $\mathcal{H}$ is the Hamiltonian, which is a constant of motion by construction due to antisymmetry of the matrix $\mathcal{B}_{i k}$.

The main feature of the non-Hamiltonian system is the failure of the Jacobi relation, which implies that the algebraic relations between phase space variables, being constructed by means of the bracket, are not invariant under time translation. This is formally a consequence of our requirement for equation (7) and the standard equation of motion to be satisfied simultaneously. By defining the Liouville operator

$$
\mathrm{i} \hat{L} A=\mathcal{B}_{i j} \frac{\partial A}{\partial x_{i}} \frac{\partial \mathcal{H}}{\partial x_{j}}=\{A, \mathcal{H}\},
$$

we obtain the generalized Liouville equation (see [17]) in the form

$$
\frac{\partial}{\partial t} \rho(q, p ; t)=-(\mathrm{i} \hat{L}+\kappa) \rho(q, p ; t)
$$

where

$$
\kappa=\sum_{i j}^{2 N} \frac{\partial \mathcal{B}_{i j}}{\partial x_{i}} \frac{\partial \mathcal{H}}{\partial x_{j}}
$$

is the phase space compressibility. For the purpose of generality we also allow it to depend on time explicitly (through $\mathcal{B}_{i j}$ ). Particularly, to determine the matrix $\mathcal{B}_{i j}$ (and therefore $\kappa$ ) it is useful to write the equation of motion in the form

$$
\dot{x}_{i}=\sum_{j=1}^{2 N} \mathcal{B}_{i j} \frac{\partial \mathcal{H}}{\partial x_{j}} .
$$

For the particular ensemble, described by equations (1)-(6), we obtain

$$
\mathcal{B}=\left(\begin{array}{cccccc}
0 & 0 & 0 & 1 & 0 & 0 \\
0 & 0 & 0 & 0 & 1 & 0 \\
0 & 0 & 0 & 0 & 0 & 1 \\
-1 & 0 & 0 & 0 & -p & 0 \\
0 & -1 & 0 & p & 0 & -p_{\eta_{1}} \\
0 & 0 & -1 & 0 & p_{\eta_{1}} & 0
\end{array}\right)
$$


so that compressibility can be calculated as

$$
\kappa=-\frac{p_{\eta_{1}}}{M_{\eta_{1}}}-\frac{p_{\eta_{2}}}{M_{\eta_{2}}} .
$$

In general for a system of $N$ identical particles (more exactly for $N$ degrees of freedom) we define the statistical time-dependent average as

$$
\langle A\rangle^{t}=\sum_{N} \int A_{N}(q, p ; t) \rho_{N}(q, p ; t) \mathrm{d} \Gamma_{N} \equiv \operatorname{Tr}\{A(q, p ; t) \rho(q, p ; t)\},
$$

where $A_{N}(q, p ; t)$ denotes the dynamic variable $A$ for a system of $N$ particles, i.e., $A\left(q_{1}, \ldots, q_{N}\right.$; $\left.p_{1}, \ldots, p_{N} ; t\right), \mathrm{d} \Gamma_{N}$ is the element of phase volume, which is assumed to be an invariant measure. For the discussion of the proper choice of the latter, see e.g. [10,11].

The time evolution of an arbitrary dynamical variable obeys the usual equation

$$
\frac{\mathrm{d} A}{\mathrm{~d} t}=\frac{\partial A}{\partial t}+\{A, \mathcal{H}\}=\frac{\partial A}{\partial t}+\mathrm{i} \hat{L} A
$$

An interesting consequence of the absence of time-translation invariance is that the average derivative of a dynamical variable is not necessarily equal to the derivative of its average. In fact, taking into account equation (8) we obtain the relation

$$
\frac{\mathrm{d}\langle A\rangle^{t}}{\mathrm{~d} t}=\int\left(\frac{\partial A}{\partial t}+\{A, \mathcal{H}\}-\kappa A\right) \rho \mathrm{d} \Gamma
$$

or, alternatively

$$
\left\langle\frac{\mathrm{d} A}{\mathrm{~d} t}\right\rangle^{t}=\frac{\mathrm{d}\langle A\rangle^{t}}{\partial t}+\kappa\langle A\rangle^{t}
$$

Equations (11) and (12) are particularly important in identifying the real measured mean quantity. Another important peculiarity of non-Hamiltonian systems follows if one is interested in the time evolution operator for the initial statistical distribution $\rho(q, p ; t)=U_{ \pm}^{(\kappa)}\left(t, t^{\prime}\right) \rho(q, p ; 0)$. Following the standard procedure [20] we obtain the time-ordered evolution operator $U_{ \pm}^{(\kappa)}\left(t, t^{\prime}\right)$ in the following form

$$
U_{ \pm}^{(\kappa)}\left(t, t^{\prime}\right)=\exp _{ \pm}\left\{-\int_{t^{\prime}}^{t}(\mathrm{i} \hat{L}(\tau)+\kappa) \mathrm{d} \tau\right\}
$$

where $\exp _{ \pm}$denote the correspondingly time-ordered exponents. We emphasize that the evolution operator in equation (13) does not describe the evolution of dynamic variables. Equation (10) gives $A(q, p ; t)=U_{ \pm}^{\dagger}\left(t, t^{\prime}\right) A\left(q, p ; t^{\prime}\right)$, where

$$
U_{ \pm}^{\dagger}\left(t, t^{\prime}\right)=U_{ \pm}^{(0) \dagger}\left(t, t^{\prime}\right)=\exp _{ \pm}\left\{\mathrm{i} \int_{t^{\prime}}^{t} \hat{L}(\tau) \mathrm{d} \tau\right\}
$$

Let us suppose that the given nonequilibrium non-Hamiltonian ensemble can be characterized by the average values $\left\langle\zeta_{n}\right\rangle^{t}$ of a finite set of dynamical variables $\left\{\zeta_{n}(t)\right\}$. Then the time evolution of the nonequilibrium macroscopic state is described by the generalized transport equations (see equations (11) and (12))

$$
\frac{\partial\left\langle\zeta_{n}\right\rangle^{t}}{\partial t}=\operatorname{Tr}\left\{\left(\dot{\zeta}_{n}-\kappa \zeta_{n}\right) \rho(t)\right\}=\operatorname{Tr}\left\{\left(\left\{\zeta_{n}, \mathcal{H}\right\}-\kappa \zeta_{n}\right) \rho(t)\right\}
$$

where the trace operation is defined in equation $(9)$. Let $\tilde{\rho}(t)$ be a trial statistical operator, such that $\left\langle\zeta_{n}\right\rangle^{t}=\operatorname{Tr}\left(\zeta_{n} \tilde{\rho}(t)\right)$, and $\operatorname{Tr}(\tilde{\rho}(t))=1$. We choose the entropy functional in the standard form [20],

$$
\tilde{S}[\tilde{\rho}(t)]=-\operatorname{Tr}(\tilde{\rho}(t) \ln \tilde{\rho}(t))-\sum_{n} \varphi_{n}(t) \operatorname{Tr}\left(\zeta_{n} \tilde{\rho}(t)\right)-\lambda(t) \operatorname{Tr} \tilde{\rho}(t),
$$


where $\varphi_{n}(t)$ and $\lambda(t)$ are the corresponding Lagrange multipliers. We have to emphasize, however, that our choice of entropy functional (16) is not rigorously justified. We just suppose it to have the structure similar to the Hamiltonian case. Alternative entropy functionals are discussed in $[10,11]$.

Varying (16) with respect to the trial distribution $\tilde{\rho}(t)$ we obtain the relevant distribution,

$$
\rho_{r}=\exp \left\{-\Phi(t)-\sum_{n} \varphi_{n}(t) \zeta_{n}\right\}
$$

where $\Phi(t)$ is the Massieu-Plank function. Here we use the notion "relevant" following Zubarev [20-22] for the distribution that maximizes the entropy functional (16) at a particular time. The relevant distribution is not yet the required nonequilibrium distribution because, in general, it does not satisfy the Liouville equation. Nevertheless, as shown below the relevant statistical distribution will serve as an auxiliary distribution to determine special solutions of the Liouville equation that describe irreversible macroscopic processes.

The Lagrange multipliers $\varphi_{n}$ are determined from the self-consistency conditions,

$$
\left\langle\zeta_{n}\right\rangle^{t}=\left\langle\zeta_{n}\right\rangle_{r}^{t} \equiv \operatorname{Tr}\left(\zeta_{n} \rho_{r}(t)\right),
$$

so that $S(t)=\Phi(t)+\sum_{n} \varphi_{n}(t)\left\langle\zeta_{n}\right\rangle^{t}$. The meaning of the nonequilibrium entropy is the same as the corresponding entropy in the Hamiltonian systems [20,21]. Contrary to the Gibbs entropy, $S(t)$ can change in time. Let us demonstrate the specific feature of this entropy in the non-Hamiltonian system by calculating the derivative

$$
\frac{\mathrm{d} S(t)}{\mathrm{d} t}=\sum_{n} \frac{\delta S(t)}{\delta\left\langle\zeta_{n}\right\rangle^{t}} \frac{\partial\left\langle\zeta_{n}\right\rangle^{t}}{\partial t}=\sum_{n} \varphi_{n} \frac{\partial\left\langle\zeta_{n}\right\rangle^{t}}{\partial t}=\sum_{n} \varphi_{n} \operatorname{Tr}\left\{\left(\dot{\zeta}_{n}-\kappa \zeta_{n}\right) \rho(t)\right\}
$$

It seems confusing that $\mathrm{d} S(t) / \mathrm{d} t \neq 0$ even for constant $\zeta_{n}$ due to the compressibility of the phase space. Of course this is misunderstanding, because the entropy and its evolution are essentially determined by the mean values of $\zeta_{n}$ and by the derivatives of their mean values. This is particularly important in applying the theory since the interpretations of the desired quantities depend on the definition of the averaging procedure.

To find the formal solution of the generalized Liouville equation (8), we assume that at some initial instant of time the distribution coincides with the relevant one (17), satisfying the selfconsistency relations (18). Following the method proposed by Zubarev [22], the retarded solution of the Liouville equation in our case is found by solving the following perturbed equation

$$
\frac{\partial \rho(t)}{\partial t}+(\mathrm{i} \hat{L}+\kappa) \rho(t)=-\epsilon\left(\rho(t)-\rho_{r}(t)\right)
$$

with $\epsilon \rightarrow 0$ in the final expressions. The formal solution of the initial Liouville equation (8) reads

$$
\rho(t)=\lim _{\epsilon \rightarrow+0} \epsilon \int_{-\infty}^{t} \mathrm{e}^{-(\epsilon+\kappa)\left(t-t^{\prime}\right)} \mathrm{e}^{-\mathrm{i}\left(t-t^{\prime}\right) \hat{L}} \rho_{r}\left(t^{\prime}\right) \mathrm{d} t^{\prime} .
$$

Integrating equation (21) by parts we arrive at

$$
\rho(t)=\rho_{r}(t)-\lim _{\epsilon \rightarrow+0} \epsilon \int_{-\infty}^{t} \mathrm{e}^{-(\epsilon+\kappa)\left(t-t^{\prime}\right)} \mathrm{e}^{-\mathrm{i}\left(t-t^{\prime}\right) \hat{L}}\left(\frac{\partial}{\partial t^{\prime}}+\mathrm{i} \hat{L}+\kappa\right) \rho_{r}\left(t^{\prime}\right) \mathrm{d} t^{\prime} .
$$

In principle equation (22) answers the question how to construct nonequilibrium distribution, which is a functional of dynamic variables and the phase space compressibility, if the system develops from a relevant state. 
Let us further write the statistical operator as a sum of relevant distribution and local-time deviation $\rho(t)=\rho_{r}(t)+\Delta \rho(t)$, so that equation (20) becomes

$$
\left(\frac{\partial}{\partial t}+\mathrm{i} \hat{L}+\kappa+\epsilon\right) \Delta \rho(t)=-\left(\frac{\partial}{\partial t}+\mathrm{i} \hat{L}+\kappa\right) \rho_{r}(t) .
$$

Then, keeping in mind that $\rho_{r}(t)$ depends on time only through the relevant variables $\zeta_{n}$, we can calculate its time derivative

$$
\begin{aligned}
\frac{\partial \rho_{r}(t)}{\partial t} & =\sum_{n} \frac{\delta \rho_{r}(t)}{\delta\left\langle\zeta_{n}\right\rangle^{t}} \frac{\partial\left\langle\zeta_{n}\right\rangle^{t}}{\partial t}=\sum_{n} \frac{\delta \rho_{r}(t)}{\delta\left\langle\zeta_{n}\right\rangle^{t}} \operatorname{Tr}\left\{\left(\dot{\zeta}_{n}-\kappa \zeta_{n}\right) \rho(t)\right\} \\
& =-\sum_{n} \frac{\delta \rho_{r}(t)}{\delta\left\langle\zeta_{n}\right\rangle^{t}} \operatorname{Tr}\left\{\zeta_{n} \frac{\partial \rho(t)}{\partial t}\right\}=-\sum_{n} \frac{\delta \rho_{r}(t)}{\delta\left\langle\zeta_{n}\right\rangle^{t}} \operatorname{Tr}\left\{\zeta_{n}(\mathrm{i} \hat{L}+\kappa) \rho(t)\right\}
\end{aligned}
$$

We define the Kawasaki-Gunton operator [23] in the standard manner

$$
\mathcal{P}_{K G} A=\rho_{r}(t) \operatorname{Tr} A+\sum_{n} \frac{\delta \rho_{r}(t)}{\delta\left\langle\zeta_{n}\right\rangle^{t}}\left\{\operatorname{Tr}\left(A \zeta_{n}\right)-\left\langle\zeta_{n}\right\rangle^{t} \operatorname{Tr} A\right\} .
$$

Using the relations $\operatorname{Tr}\{\mathrm{i} \hat{L} \rho(t)\}=0, \operatorname{Tr}\{\dot{\rho}(t)\}=-\langle\kappa\rangle^{t}$, one can prove that the projection operator (21) conserves its features in the non-Hamiltonian case, namely $\mathcal{P}_{K G}(t) \rho(t)=\rho_{r}(t), \mathcal{P}_{K G}(t) \dot{\rho}(t)=$ $\dot{\rho}_{r}(t)$. Equation $(24)$ can now be rewritten in the projected form

$$
\frac{\partial \rho_{r}(t)}{\partial t}=-\mathcal{P}_{K G}(t)(\mathrm{i} \hat{L}+\kappa) \rho(t) .
$$

Inserting equation (26) into equation (23) and integrating over time, we obtain a solution for $\Delta \rho(t)$ as follows

$$
\Delta \rho(t)=-\int_{-\infty}^{t} \mathrm{e}^{-\epsilon\left(t-t^{\prime}\right)} U_{r}^{(\kappa)}\left(t, t^{\prime}\right) \mathcal{Q}_{K G}\left(t^{\prime}\right)(\mathrm{i} \hat{L}+\kappa) \rho_{r}\left(t^{\prime}\right) \mathrm{d} t^{\prime},
$$

where $\mathcal{Q}_{K G}=1-\mathcal{P}_{K G}$, and (compare with equation (13))

$$
U_{r}^{(\kappa)}\left(t, t^{\prime}\right)=\exp \left\{-\int_{t^{\prime}}^{t} \mathcal{Q}_{K G}(t)(\mathrm{i} \hat{L}+\kappa) \mathrm{d} \tau\right\} .
$$

Equation (27) allows us to express the right-hand side of equation (15) in terms of relevant distribution, i.e. in terms of variables $\left\langle\zeta_{n}\right\rangle^{t}$. So we get the closed set of generalized transport equations for observables.

To obtain more transparent equations we define the entropy operator

$$
\hat{S}(t)=\Phi(t)+\sum_{n} \varphi_{n}(t) \zeta_{n}(t),
$$

so that $\rho_{r}(t)=\exp \{-\hat{S}(t)\}$. Introducing the Mori projection operator [24]

$$
\mathcal{P}_{M}(t) A=\langle A\rangle_{r}^{t}+\sum_{n} \frac{\delta\langle A\rangle_{r}^{t}}{\delta\left\langle\zeta_{n}\right\rangle^{t}}\left(\zeta_{n}-\left\langle\zeta_{n}\right\rangle^{t}\right),
$$

which satisfy the identities $\mathcal{P}_{M}^{2}(t)=\mathcal{P}_{M}(t), \mathcal{P}_{M}(t) \zeta_{n}=\zeta_{n}$, one can prove the relation

$$
\mathcal{Q}_{K G}(t)\left\{(\hat{i} \hat{L}+\kappa) \rho_{r}\right\}=\mathcal{Q}_{M}(t)\{\kappa-\mathrm{i} \hat{L} \hat{S}\} \rho_{r}
$$


where $\mathcal{Q}_{M}(t)=1-\mathcal{P}_{M}(t)$ is complementary to the Mori projector (29). Now the generalized transport equation (15) reads

$$
\frac{\partial}{\partial t}\left\langle\zeta_{n}\right\rangle^{t}=\frac{\partial}{\partial t}\left\langle\zeta_{n}\right\rangle_{r}^{t}+\operatorname{Tr}\left\{\mathcal{Q}_{M}(t)\left(\dot{\zeta}_{n}-\kappa \zeta_{n}\right) \Delta \rho\right\},
$$

where $\Delta \rho$ should be taken from equation (27). It is useful to define new dynamical variables (generalized fluxes)

$$
\mathcal{I}_{n}(t)=\mathcal{Q}_{M}(t)\left(\dot{\zeta}_{n}-\kappa \zeta_{n}\right),
$$

which are orthogonal to the space of relevant variables in the sense that $\mathcal{P}_{M} \mathcal{I}_{n}(t)=0$. The variables $\mathcal{I}_{n}(t)$ determine the effects of macroscopic degrees of freedom of the evolution on the nonequilibrium state of the non-Hamiltonian system described by a set of relevant variables and a given phase space compressibility $\kappa$.

Incorporating equations (27), (30), and (31) we obtain the final result for the generalized transport equations

$$
\frac{\partial}{\partial t}\left\langle\zeta_{n}\right\rangle^{t}=\frac{\partial}{\partial t}\left\langle\zeta_{n}\right\rangle_{r}^{t}+\sum_{n} \int_{-\infty}^{t} \mathrm{e}^{-\epsilon\left(t-t^{\prime}\right)} \mathcal{D}_{m n}\left(t, t^{\prime}\right) \varphi_{n}\left(t^{\prime}\right) \mathrm{d} t^{\prime}+\int_{-\infty}^{t} \mathrm{e}^{-\epsilon\left(t-t^{\prime}\right)} \mathcal{F}_{m n}\left(t, t^{\prime}\right) \mathrm{d} t^{\prime},
$$

where

$$
\mathcal{D}_{m n}\left(t, t^{\prime}\right)=\operatorname{Tr}\left\{\mathcal{I}_{m}(t) U_{r}^{(\kappa)}\left(t, t^{\prime}\right) \mathcal{I}_{m}\left(t^{\prime}\right) \rho_{r}\left(t^{\prime}\right)\right\}
$$

are the normal generalized kinetic coefficients, and

$$
\mathcal{F}_{m n}\left(t, t^{\prime}\right)=\operatorname{Tr}\left\{\mathcal{I}_{m}(t) U_{r}^{(\kappa)}\left(t, t^{\prime}\right) \mathcal{Q}_{M}\left(t^{\prime}\right) \kappa\left(\sum_{n} \varphi_{n} \zeta_{n}-1\right) \rho_{r}\left(t^{\prime}\right)\right\}
$$

are the effective forces determining the anomalous contribution into the system evolution associated with nonlinear phase space compressibility.

The last term in equation (32) is associated with the absence of time-translation invariance of the process due to non-Hamiltonian behavior, while the second term is due to the nonequilibrium process itself. It does not vanish even when $\kappa=0$, and coincides with the well-known result for Hamiltonian ensembles. These terms demonstrate the general structure of the solution and clearly show the contributions from two physically different processes. In practice equations (33) and (34) can be used with standard simplifications for particular systems. However, we should note that our formalism could be safely used if the reference system is too close to equilibrium. In particular, the choice of the entropy functional in equation (22) is unlikely to be well justified.

In conclusion, we have proposed a possible realization of the nonequilibrium statistical mechanics for ensembles of particles obeying non-Hamiltonian dynamics. Starting with the nonHamiltonian Poisson bracket (7) and the equations of motion in standard form, we derived the basic relations for time-dependent dynamical variables with conserved Hamiltonian. Introducing the reduced description method in analogy with Hamiltonian dynamics we found the retarded solution (22) of the generalized Liouville equation (8). We emphasize, however, that equation (22) is a specific solution, rather than general, and it describes the evolution from a specific auxiliary relevant distribution (this is reflected in the introduction of parameter $\epsilon$ ). Using the projection operator technique we obtained the generalized transport equations (32), which contain two physically different contributions due to the statistical irreversibility (33) and the time-irreversibility (34) associated with non-Hamiltonian features of the system.

\section{Acknowledgements}

The research is supported by the NSF career award and the ACS petroleum research fund. 


\title{
References
}

1. Ashurst W.T., Hoover W.J., Phys. Rev. Lett., 1973, 31, 206.

2. Anerson H.C., J. Chem. Phys., 1983, 72, 2384.

3. Evans D.J., J. Chem. Phys., 1983, 78, 3297.

4. Nosé S., Mol. Phys., 1984, 52, 255.

5. Hoover W.G., Phys. Rev. A, 1985, 31, 1695.

6. Martyna G.L., Klein M.L., Tuckerman M., J. Chem. Phys., 192, 97, 2635.

7. Cao J.S., Voth G.A., J. Chem. Phys., 1996, 105, 6856.

8. Klein P., Modell. Simul. Mater. Sci. Eng., 1998, 6, 405.

9. Yang S.L., Cao J.S., J. Chem. Phys., 2002, 117, 10996.

10. Tuckerman M.E., Yi Liu, Ciccotti G., Martyna G.J, J. Chem. Phys., 2001, 115, 1678.

11. Tuckerman M.E., Mundy C.J., Martyna G.J., Europhys. Lett., 1999, 45, 149.

12. Tully J.C., Faraday Discussions, 1998, 110, 407.

13. Kapral R., Ciccotti C., J. Chem. Phys., 1999, 110, 8919.

14. Nielsen S., Kapral R., Ciccotti C., J. Chem. Phys., 2000, 112, 6543.

15. Nielsen S., Kapral R., Ciccotti C., J. Chem. Phys., 2000, 115, 5805.

16. Burant J.C., Tully J.C., J. Chem. Phys., 2000, 112, 6097.

17. Sergi A., Ferrario M., Phys. Rev. E., 2001, 64, 056125.

18. Sergi A., Phys. Rev. E., 2003, 67, 021101.

19. Morrison P.J., Rev. Mod. Phys., 1998, 70, 467.

20. Zubarev D. Nonequilibrium Statistical Thermodynamics. Nauka, Moscow, 1971.

21. Zubarev D., Morozov V., Röpke G. Statistical Mechanics of Nonequilibrium Processes. Akademie Verlag, Berlin, 1996.

22. Zubarev D. Modern Methods of Statistical Theory of Nonequilibrium Processes, in Science and Technology Report, Modern Problems of Mathematics. VINITI, Moscow, 1996.

23. Kawasaki K., Gunton J.D., Phys. Rev. A, 1973, 8, 2048.

24. Mori H., Prog. Theor. Phys., 1965, 33, 423.

25. Sergi A., Phys. Rev. E, 2004, 69, 021109.

\section{Кінетична теорія негамільтонових статистичних ансамблів}

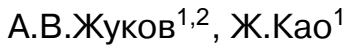 \\ 1 Хімічний факультет, Масачусецький технологічний інститут, Кембрідж, МА 02139,США \\ 2 Хімічний факультет, університет західного Онтаріо, Лондон, Онтаріо, N6A 5B7, Канада \\ Отримано 21 вересня 2005 р.
}

Розвинуто метод нерівноважного статистичного оператора для ансамблів частинок, які підкоряються негамільтоновим рівнянням руху в класичному фазовому просторі. Основні наслідки ненульової стисливості фазового простору вивчаються на мові часовозалежних динамічних величин.

Ключові слова: негамільтонові системи, нерівноважний статистичний оператор, формалізм Зубарєва, молекулярна динаміка

PACS: $05.20 .-y, 05.20 . \mathrm{Gg}, 82.20 .-w$ 
